\title{
Voice Performance Analysis using Voice Codec by Packet Fragmentation and Contention Free periods in Wireless Networks
}

\author{
${ }^{1}$ Fakrudeen, M., ${ }^{1}$ Yousef, S., ${ }^{2}$ Tapaswi, S., ${ }^{2}$ Patnaik, K. K., and ${ }^{1}$ Cole, M \\ ${ }^{1}$ Anglia Ruskin University, Chelmsford, UK \\ \{Sufian.Yousef@anglia.c.uk\} \\ ${ }^{2}$ ABV-Indian Institute of Information Technology and Management, Gwalior, India
}

\begin{abstract}
The admission control is required to maintain the established route between the source and the destination in the wireless network. To maintain the stability of the route, the wireless channel parameters has to be adopted appropriately. Thus, this study analyzes the wireless access medium parameter through Direct Coordination (DCF) and Point-to-Point Coordination (PCF) method. The packets are fragmented in DCF and contention free period interval are adjusted to study the QoS parameters for various VOIP codec using OPNET simulation tool. The result shows that packet fragmentation to 256 bytes and contention free period for $20 \mathrm{msec}$ improves QoS for G.729 for Voice traffic.
\end{abstract}

Keywords: VOIP, Codec, QoS , Admission Control and MANET

\section{Introduction}

Voice over Internet Protocol (VOIP) delivers voice over the internet using Internet Protocol (IP). Currently, VOIP related applications such as Yahoo messenger, Skype, Viber, and Tango provide free calls with good quality. While transmitting the voice, these applications convert the analog voice signal into the digital format before compression and transmitted as IP packets after encoding over IP network. During receiving the voice, using Digital to Analog Converter (DAC), the digital format is converted to analog format by reassembling the IP packets for further processing [1].

Wireless network is a collection of mobile nodes connected dynamically with or without any infrastructure such as base station. The ad hoc routing protocols such as Ad hoc On-demand distance Vector(AODV), Dynamic Source Routing(DSR) and Open Link Source Routing(OLSR) play a vital role in creating the route to communicate with different nodes. However, due to the mobility of the nodes, link stability between the nodes is poor which may lead to congestion.

In wireless communication, when the number of VOIP calls exceeds the capacity, the Quality of Service (QoS) deteriorates for each call. Thus, voice quality becomes the point of concern. Consequently, it is necessary to have admission control to protect the voice quality of the existing calls. The admission control differs widely for Ethernet wired and IEEE 802.11 wireless connections. In a wired environment, the 
admission control depends on end-to-end QoS. But in the wireless communication, the admission control should be applied between the access point (gateway) and the wireless stations.

As a result, for effective admission control, it is necessary to analyze the wireless channel settings for voice quality such as Voice data packet rate and collision rate depending on the bandwidth of the wireless channels. By accurately adjusting the wireless channel parameters, the voice quality can be enhanced.

Wi-Fi networks possess dispute regarding the quality of Voice communication. In comparison with wired networks, the throughput of wireless communication is much less. Wi-Fi uses Carrier Sense Multiple Access and Collision Avoidance (CSMA/CA) as the accessing medium which spent most of the time in collision avoidance which causes delay and degradation in voice quality. In addition, the channel utilization is very much limited near to the access point region in multi-hop Wi-Fi network.

In IEEE 802.11 standards, call drop or call jitter can occur in heavy traffic which affects the voice quality of Voice calls. This demands the use of a through and precise use of wireless channel which helps in the reduction of call drops and call jitter.

The review of the literature shows that various studied has been performed on Quality of Service (QoS) parameters of Voice in both wired Ethernet Lan and Wireless LAN. The researchers compare the performance both using QoS parameters such as throughput and average delay [2]. Anand et al studied VOIP traffic using the various routing protocol. Simulation using OPNET shows that Open Link State Routing (OLSR) protocol performed better than the other routing protocol in terms of delay and throughput [3]. Anouri et al analyses the QoS parameter for WiMAX network over various service classes [4]. [5] discussed inter and intra mobility for VOIP traffic in wireless LAN during handoff mechanism.

Atitur et al derived the analytical model for calculating Voice call capacity for single and multiple hops using IEEE $802.11 \mathrm{~g}$ wireless LAN network[6]. The result shows that the number of hops has effects on the call quality. Hussein et al studied VOIP traffic using different Queueing algorithm [7]. The result reveals that Weight Fair Queuing algorithm and priority queue are the best queuing algorithm which improves QoS for VOIP. The main limitation of QoS in wireless communication is studied in comparison with the wired network by keeping Voice as multimedia measurement.

However, none of the studies predicts QoS for wireless communication in Voice traffic using various audio codec schemes for the various wireless accessing medium such as DCF and PCF.

\section{Voice Codecs, DCF and PCF}

A call which uses Voice infrastructure has to undergo two different stages. In the first stage in transmitter node, the standard analog signal is converted into digital format (encoding) to transmit through the internet. During the second stage at the receiver end, the digital format is converted back to analog format (decoding). To perform encoding everything, waveform algorithm such as PCM (Pulse Code Modulation) and AD PCM(Adaptive Differential PCM) is applied. To encode partially, the source 
algorithm such as CS-ACELP (Conjugate Structure Algebraic Code-Excited Linear Prediction) and LDCELP (Low Delay-Code Excited Linear Prediction) is used.

There are different Voice codecs are available based on selected sampling, data rate, and compression implementation. The features of Voice codecs used in this research is summarized in Table 1.

Table 1. Voice Codec Features [8]

\begin{tabular}{|c|c|c|c|c|}
\hline VOIP Codec & Algorithm & $\begin{array}{l}\text { Codec } \\
\text { Delay }(\mathrm{ms})\end{array}$ & $\begin{array}{l}\text { Bit } \\
\text { Rate(Kbps) }\end{array}$ & Comments \\
\hline G.711 & $\overline{\mathrm{PCM}}$ & 0.375 & 64 & $\begin{array}{lr}\text { Required very } & \text { low } \\
\text { processor } & \\
\text { requirements } & \text { and } \\
\text { delivers precise } \\
\text { speech transmission }\end{array}$ \\
\hline G.723.1 & $\begin{array}{l}\text { Multipulse maximum } \\
\text { likelihood } \\
\text { quantization/algebra c- } \\
\text { code excited linear } \\
\text { prediction }\end{array}$ & 97.5 & 5.3 & $\begin{array}{l}\text { Required lot of } \\
\text { processing power. } \\
\text { High quality audio is } \\
\text { delivered using high } \\
\text { compression }\end{array}$ \\
\hline G.729 & CS-ACELP & 35 & 8 & $\begin{array}{l}\text { Error tolerant and } \\
\text { excellent bandwidth } \\
\text { utilization }\end{array}$ \\
\hline
\end{tabular}

QoS of wireless networks is challenged by the interferences, hidden node problem, multipath propagation, handoff, propagation delay and medium access method. IEEE 802.11 designed for wireless media, used the DCF (Distributed Coordination Function) as the mechanism for accessing the wireless medium. However, it could also implement PCF (Point Coordination Function) on the top of DCF optionally.

DCF uses CSMA/CA to share the wireless medium between the multiple nodes. Still, it also uses RTS/CTS (Request to Send/ Clear to Send) as optional. In DCF, VOIP traffic is not prioritized when many nodes start sending data at the same time which causes the collision. Thus, it allows only one station to access the medium. If it has low bit rate, then it takes longer time for each node to send its traffic. In DCF, the QoS cannot be guaranteed.

Fragmentation threshold is a technique in DCF used to fragment the packet. If the packet size exceeds the threshold, the packets are fragmented. The number of fragments will depend on fragmentation threshold and packet size. The fragmentation of larger sized packets improves the transmission between the source and destination.

PCF is used as optionally for transmitting traffic which is time-sensitive. It will be functional in infrastructure mode where access point act as the coordinator, controlling the beacon interval (approximately 0.1 seconds) to allow the nodes to transmit.

PCF defines two periods namely Contention Free period (CFP) and Contention period (CP). In CPF, the access point sends packets (Contention Free Pool) to all the nodes for sending their traffic. However, in CP mode, it simply uses DCF. Therefore, PCF allows all the nodes to transmit their packets at regular interval with time delay 
and hence it can be termed as contention free protocol. Due to this fact, the PCS provides better management of QoS.

In this study, Voice traffic is adjusted with fragmentation threshold and various contention free (CF) interval to enhance the QoS of VOIP traffic. The following parameters are studied to understand the QoS rendered by VOIP traffic.

MOS: It is Mean Opinion Score which indicates the quality of Voice. MOS is expressed from 1 to 5,1 being worst and 5 being the best.

Jitter : it is termed as packet delay variation. If two consecutive packets leave at the time stamp of T1 and T2 at the source node and reach the destination at the time stamp of T3 and T4 respectively. Then Jitter is calculated by:

$$
\text { Jitter }=(\mathrm{T} 4-\mathrm{T} 3)-(\mathrm{T} 2-\mathrm{T} 1)
$$

Packet End-to-End Delay: This delay caused by the packet to reach from source to destination. It is calculated by the formula:

$$
\text { Delay }=\text { Network_Delay }+ \text { Encoding_Delay }+ \text { Decoding_Delay }+
$$

Compression_Delay + Decompression_Delay + Dejitter_Buffer_Delay

Traffic Sent (bytes/sec): It is the average number of bytes submitted per second by all voice applications to the transport layers in the network.

Traffic Received (bytes/sec): It is the average number of bytes forwarded per second by the transport layers in the network to all Voice applications.

\section{Simulation Configuration}

To study the performance analysis of Voice traffic under various Codec schemes using DCF and PCF mode, extensive simulation is conducted in OPNET 18.5.

\subsection{Network Topology}

For the network model, 3 Basic Service Set (BSS) is considered. Each BSS includes one access point and 5 wireless stations. Each BSS has a radius of $1 \mathrm{Km}$ in which nodes are placed in the random fashion. All 3 access points are connected to switch which in term is connected to VOIP Server.

\subsection{Wireless Setup and Scenarios}

The Voice application is configured for IP Telephony with 0.02 seconds for compression delay and decompression delay each. The VOIP traffic is considered with and without suppression The Wireless LAN attributes are shown in Table 2.

Table 2. Wireless LAN parameters used in Simulation.

\begin{tabular}{ll}
\hline Parameter & Value \\
\hline Physical Characteristics & Direct Sequence \\
Data Rate & $11 \mathrm{Mbps}$ \\
Transmit Power & $0.0064 \mathrm{~W}$
\end{tabular}




\begin{tabular}{ll} 
Power Threshold & $-95 \mathrm{db}$ \\
Short Retry Limit & 7 \\
Power Threshold & 4 \\
\hline
\end{tabular}

For DCF, Fragmentation threshold is configured for each VOIP codec. Thus, 9 scenarios are conducted with parameters summarized in Table 3.

Table 3. DCF parameter for VOIP traffic.

\begin{tabular}{lll}
\hline Mode & $\begin{array}{l}\text { Fragmentation Threshold } \\
\text { (bytes) }\end{array}$ & VOIP Codec \\
\hline DCF & None, 256 and 1024 & G.711, G.723 and G.729 \\
\hline
\end{tabular}

For PCF, the Contention Free Period (CFP) is adjusted for every data rate and VOIP Codec. Thus, 12 scenarios are conducted with parameters summarized in Table 4.

Table 4. PCF parameter for VOIP traffic.

\begin{tabular}{llll}
\hline Mode & CFP (msec) & Data Rate(Mbps) & VOIP Codec \\
\hline PCF & 20,40 & 1,11 & G.711, G.723 and G.729 \\
\hline
\end{tabular}

\section{Result and Analysis}

\subsection{Fragmentation Threshold}

The performance of Voice traffic is studied by altering the fragmentation threshold value to none, 256 and 1024 bytes. The result is summarized in Table 3.

Table 3. Results of Fragmentation threshold in DCF mode

\begin{tabular}{lll}
\hline Parameter & $\begin{array}{l}\text { Fragmentation } \\
\text { Threshold(Bytes) }\end{array}$ & Results \\
\hline MOS & None & $\begin{array}{l}\text { G729 has higher MOS than G711 } \\
\text { and G723 } \\
\text { MOS unaffected with Codec and } \\
\text { maintain the same. }\end{array}$ \\
\hline End-to-End Delay & None & $\begin{array}{l}\text { G711 have higher packet end-to-end } \\
\text { delay compared to G723 and G729 } \\
\text { G711 have higher packet end-to-end } \\
\text { delay compared to G723 and G729 }\end{array}$ \\
& None & $\begin{array}{l}\text { G711 has higher Jitter at the initial } \\
\text { stage. When the traffic increases, } \\
\text { G729 Jitter increases and G711 } \\
\text { decreases } \\
\text { G729 has higher Jitter than G711 }\end{array}$ \\
& 256 and 1024 &
\end{tabular}


and G723

\begin{tabular}{lrll}
\hline Traffic & Sent and None & Traffic Sent : G711 sent higher \\
Traffic Received & & traffic than G723 and G729 \\
(Bytes/Sec) & & Traffic Received : G711 receives \\
& higher traffic than G723 and G729 \\
& Traffic Sent : G711 sent higher \\
& traffic than G723 and G729 \\
& & Traffic Received : G711 receives \\
& higher traffic than G723 and G729 \\
\hline
\end{tabular}

MOS: It describes the voice perception quality. G729 has higher MOS than G711 and G723. However, the packet fragmentation does not impact MOS of all Codec.

End-to-End Delay. The fragmentation threshold significantly improves the QoS of Voice in terms of packet end-to-end delay. G.729 has less packet size and higher bit rate which significantly has less delay compared to G.711 and G.723, which has higher packet size and lower transform rate.

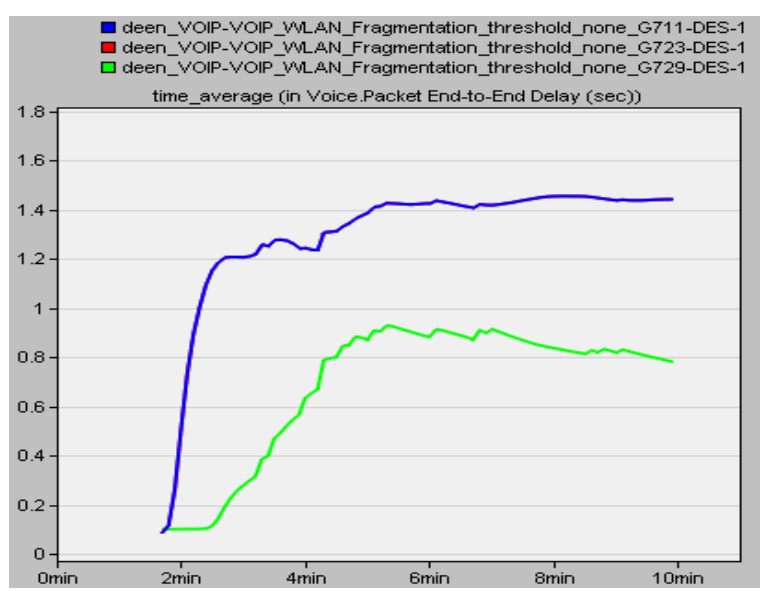

Fig. 1. Packet End-to-End Delay without fragmentation threshold under various Codecs

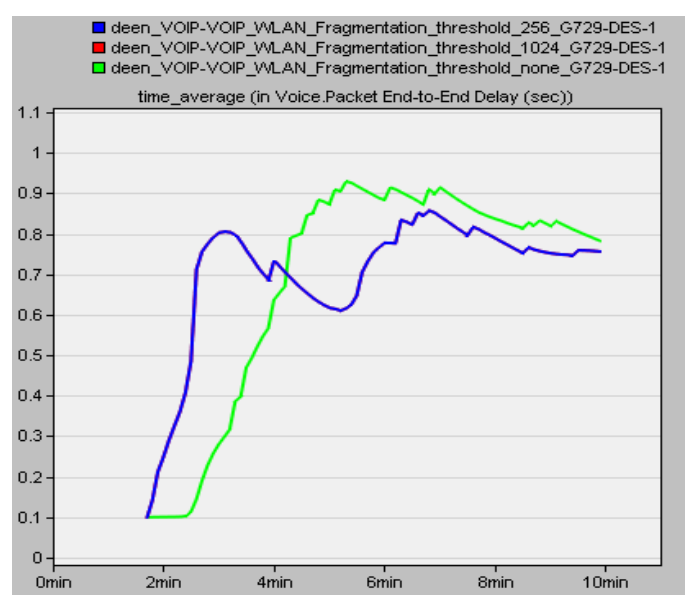

Fig. 2. Packet End-to-End Delay with fragmentation threshold under constant Codec 


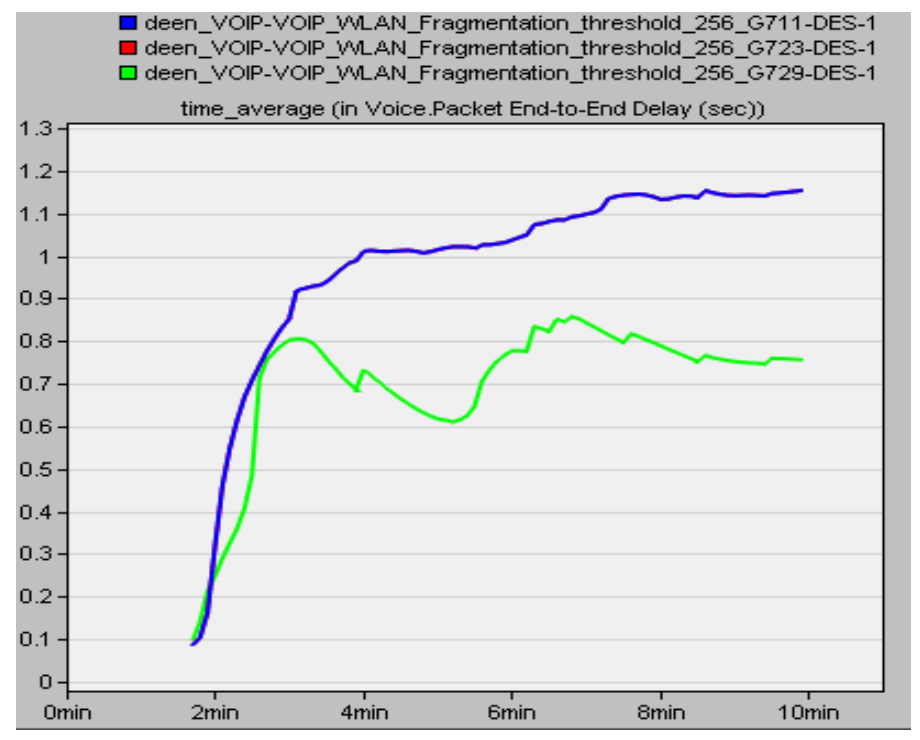

Fig. 3. Packet End-to-End Delay with single fragmentation threshold under various Codecs

Jitter: Due to complexity in packet arrival at the different level of time, the high value of jitter makes voices much difficult to understand. The use of G.711 will make jitter less for the best performance in the wireless network.

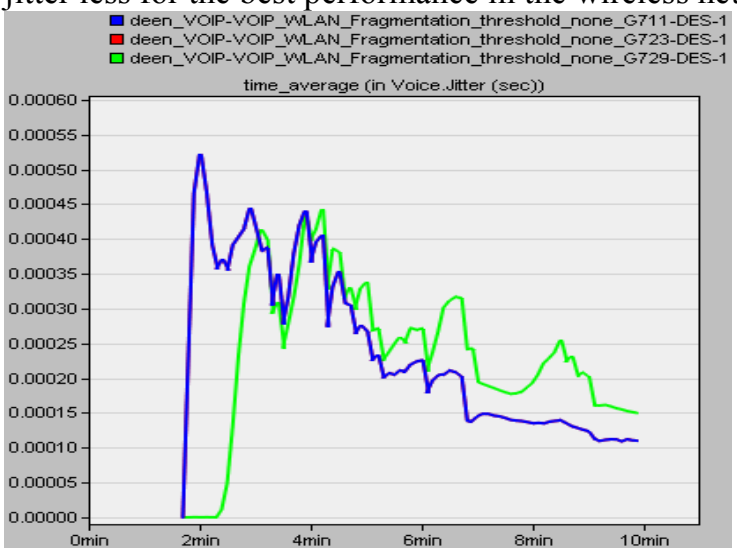

Fig. 4. Voice Jitter without fragmentation threshold under various Codecs

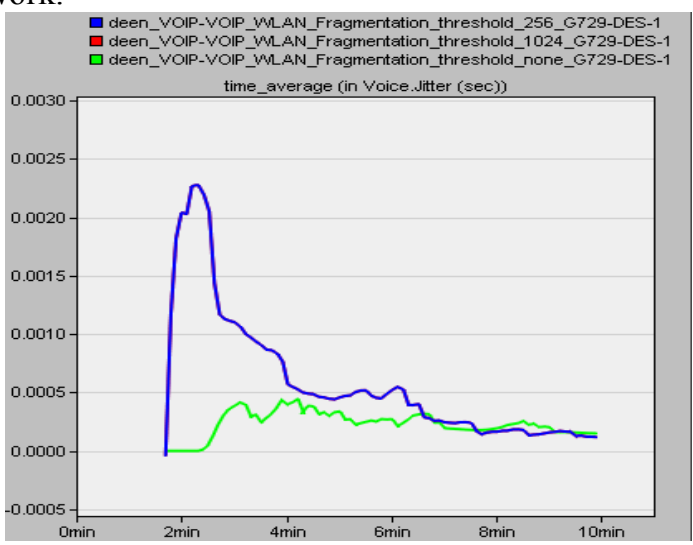

Fig. 5. Voice Jitter with fragmentation threshold under constant Codec 


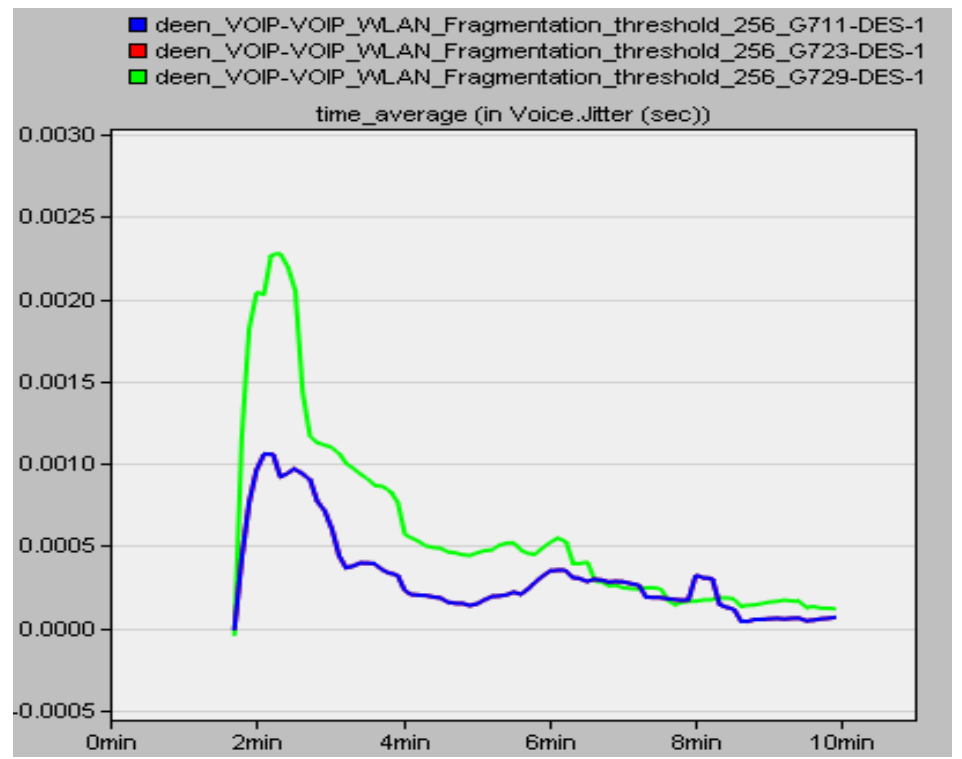

Fig. 6. Voice Jitter with single fragmentation threshold under various Codecs

Traffic Sent and Traffic Received (bytes/sec) : On total, to have an efficient network the traffic of receiving and sending must be similar. The traffic is less in G.729 when compared to G.711 and G.723. This analysis indicates that G.729 has less noise compared to other VOIP codecs. Thus, it is more efficient to use G.729 than other codecs in terms of traffic.

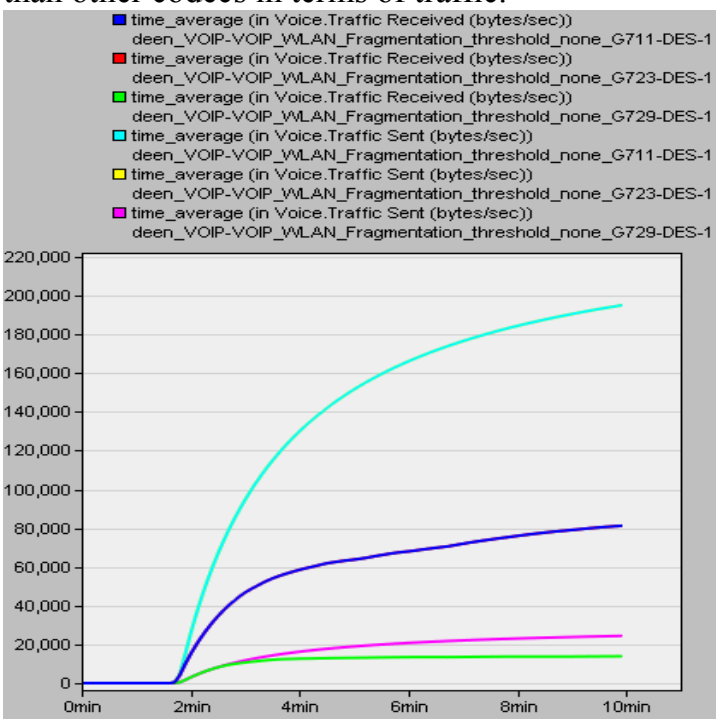

Fig. 7. Traffic Sent and Traffic Received without fragmentation threshold under various Codecs

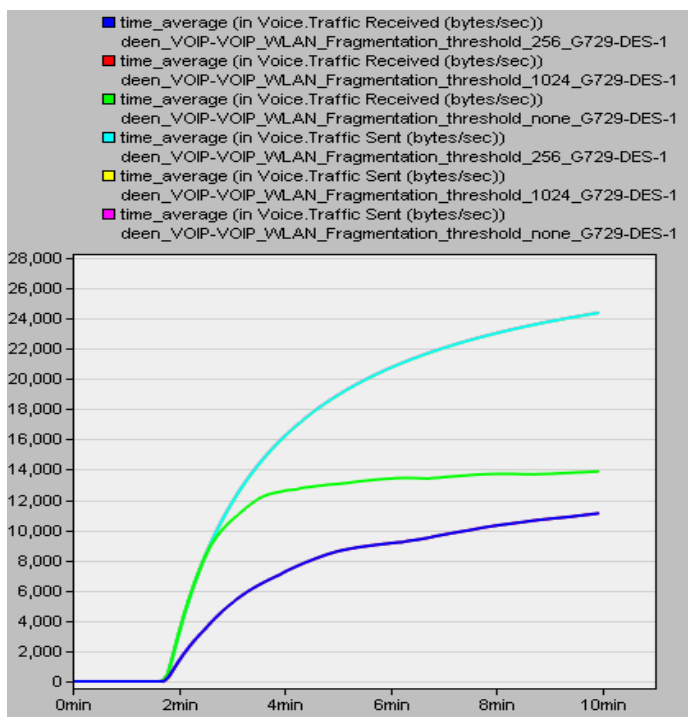

Fig. 8. Traffic Sent and Traffic Received with fragmentation threshold under constant Codec 


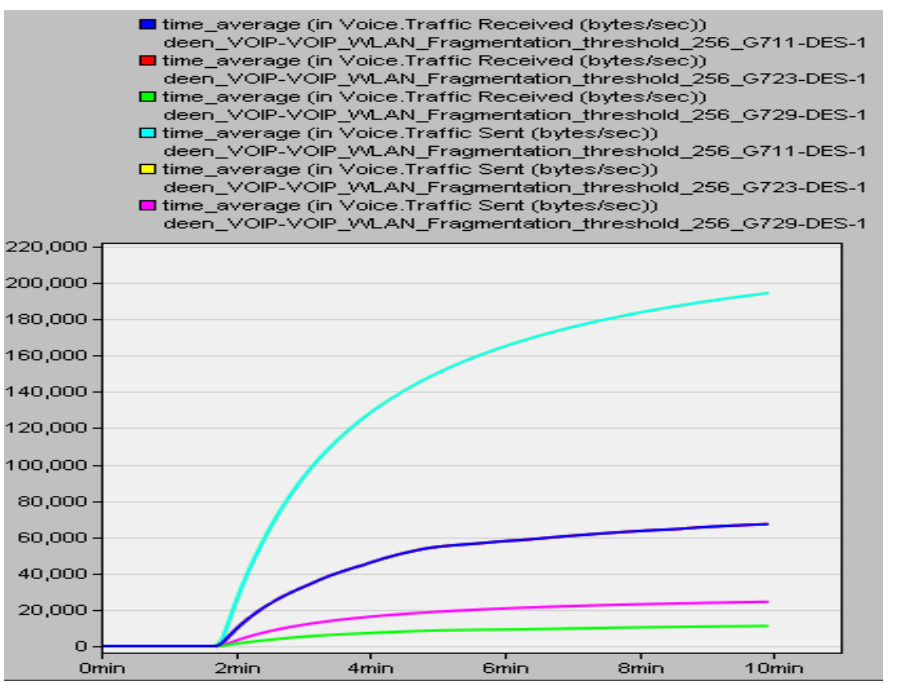

Fig. 9. Traffic Sent and Traffic Received with single fragmentation threshold under various Codecs

\subsection{PCF mode for various VOIP Codec}

With PCF mode enabled, the Voice traffic is analyzed for different codec by altering CFP to $40 \mathrm{~m} \mathrm{sec}$ and data rate to $11 \mathrm{Mbps}$. G.729 has less end-to-end delay compared to other codecs.

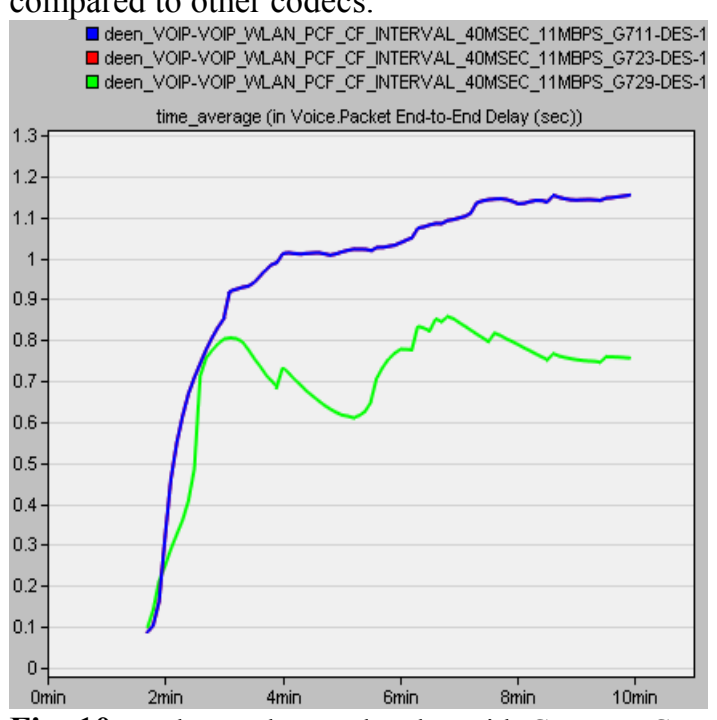

Fig. 10. Packet End-to-End Delay with Constant CFP and data rate under various Codecs

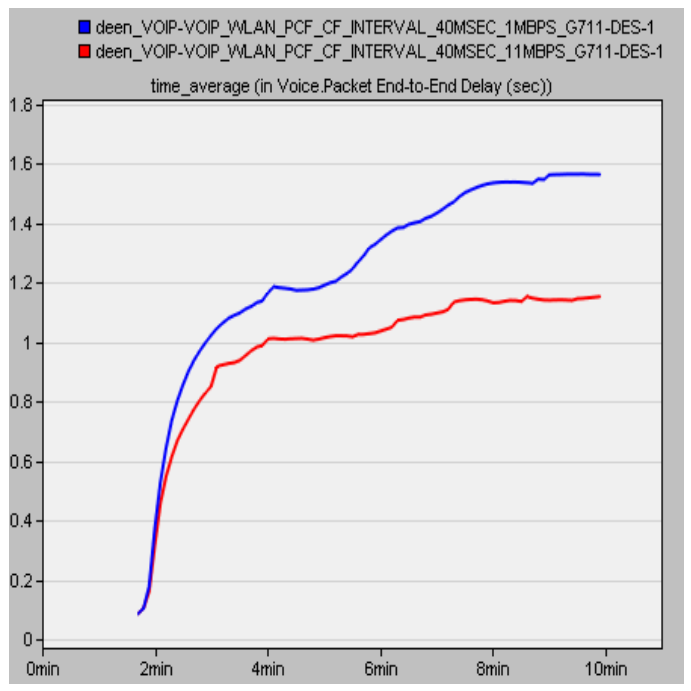

Fig. 11. Packet End-to-End Delay with Constant CFP and variable data rate under constant Codec 
Under single Codec, with constant CFP period of $40 \mathrm{msec}$, the study has been analyzed using data rates for 1 and $11 \mathrm{Mbps}$. The result shows the end-to-end delay is less for the data rate of $11 \mathrm{Mbps}$.

\section{Conclusion}

Despite the establishment of a route to transform the Voice packets from source to destination by the routing protocol, maintaining the route stability is a daunting task due to the variety of reason. One of the reason is the wireless channel settings where VOIP packets propagate.

The proposed scheme exploits the channel quality by fragmenting bigger Voice packets and adjusting the contention period to different values. The experiment is analyzed with different compression/ decompression technique of Voice codecs such as G.711, G.723, and G.729.

The scenarios were examined with 256 and 1025 bytes as fragmentation threshold. In addition, the experiments were also conducted with 20 and $40 \mathrm{msec}$ contention period for the wireless channel. The Voice traffic is analyzed with QoS parameters such as MOS, End-to-End delay, Traffic received and Traffic sent.

The study result clearly indicates that VOIP performance is good by fragmentation than without fragmentation of VOIP packets. The contention free period worked well with an increase in the data rate. On the whole, fragmentation and contention free period performed well in G.729 compared to other VOIP codecs in terms of end-toend delay, traffic received and traffic sent except Jitter.

\section{Acknowledgment}

The authors thank the UK-India Education and Research Initiative (UKIERI) for sponsoring this research project (UGC-2013-14/037) between Anglia Ruskin University in UK and the ABV-Indian Institute of Information Technology and Management in India for two years.

\section{References}

1. Jung, Y., \& Manzano, C. (2014). Burst packet loss and enhanced packet loss-based quality model for mobile voice-over Internet protocol applications. IET Communications, 41-49.

2. Neupane, K., Kulgachev, V., Elam, A., Vasireddy, S., \& Jasani, H. (2011). Measuring the Performance of VoIP over Wireless LAN. SIGITE'11 (pp. 269-274). New York, USA: ACM.

3. Anand, S., Vaidya, R., \& Velmurugan. (2011). Performance Analysis of VoIP Traffic using various Protocols and Throughput enhancement in WLANs. International Conference on Computer, Communication and Electrical Technology - ICCCET 2011, (pp. 176-180).

4. Anouari, T., \& Haqiq, A. (2012). Performance Analysis of VoIP Traffic in WiMAX using Various Service Classes. International Journal of Computer Applications, 2934. 
5. Lakas, A. (2007). Experimental Analysis of VoIP over Wireless. Local Area Networks. Journal of Communications, Vol 2-4, 3-9.

6. Siddique, M., Kamruzzaman, J., \& Hossain, M. (2014). An Analytical Approach for Voice Capacity Estimation Over WiFi Network Using ITU-T E-Model. IEEE TRANSACTIONS ON MULTIMEDIA, VOL. 16, NO. 2, 360-372.

7. Mohammed, H., Ali, A., \& Mohammed, H. (2013). The Affects of Different Queuing Algorithms within the Router on QoS VoIP application Using OPNET. International Journal of Computer Networks \& Communications (IJCNC) Vol.5, No.1, 117-124.

8. Alsahlany, A. (79-89). Performance Analysis of VOIP Traffic over Integrating Wireless LAN and WAN Using Different Codecs. International Journal of Wireless \& Mobile Networks (IJWMN) Vol. 6, No. 3, 2014. 